\title{
ELABORACIÓN DE UN CONCEPTO: VEJEZ
}

Elizabeth Murrain Knudson*

\section{Resumen}

A través de la didáctica y la dialéctica, la expresión y el manejo de los conceptos son elementos fundamentales del proceso de enseñanza y aprendizaje, ya que su claridad debe ser tal que al comunicarse los profesionales entre sí, estos representen lo mismo en sus procesos cognitivos. Esta claridad conceptual es fundamental en el desempeño clínico y académico, es por esta razón que existe una inagotable lista de palabras definidas en los glosarios de la salud. Al ver que una palabra tan cotidiana como vejez tiene significados tan variados entre nuestras enfermeras y estudiantes de enfermería, surgió una inquietud académica que tiene repercusión directa en el cuidado de las personas viejas en nuestra sociedad colombiana, aprovechando y siguiendo con rigor los pasos para la elaboración de un concepto dado por Cutcliffe J. and McKenna H. The Essential Concepts of Nursing (2005). Deseo socializar este ejercicio académico teniendo en cuenta la reinauguración de la especialización en gerontología en la facultad de enfermería de la Fundación Universitaria de Ciencias de la Salud.

Palabras clave: vejez, elaboración de un concepto, cuidado de enfermería.

\section{Abstract}

Management and expression of concepts through didactics and dialectics are essential elements of the teaching and learning processes. Clear concepts are needed to enable good intercommunication among professionals allowing a similar representation of these concepts in their cognitive processes. Clear concepts are critical in clinical and academic performance, accounting for a vast number of words to be listed in health glossaries. As we became aware that everyday-used words such as old age have such distinct meanings within our nurses and nursing students, we developed an academic initiative with direct repercussion on elderly care in Colombia using and strictly following the steps for concept building stated by Cutcliffe J. and McKenna H. in The Essential Concepts of Nursing (2005). I wish to introduce this academic experience and encourage its inclusion in the Gerontology Specialization Program of the Nursing School of the Health Sciences University Foundation, provided it has been re-opened.

Key words: old age, building a concept, nursing care.

\section{Presentación}

Una vez efectuada la revisión de la literatura respecto al tema de "vejez y enfermedad crónica, vejez y discapacidad, vejez y dependencia, vejez y funcionalidad", desde octubre 24 hasta noviembre 20 de 2006 ingresé a las bases de datos CINAHL, OVID,

Fecha recibido: agosto 8 de 2007- Fecha aceptado: octubre 30 de 2007

* Enfermera egresada de la Universidad Nacional de Colombia. Especialista en auditoría en salud, diplomada en docencia universitaria y en epidemiología. Magistrado del Tribunal de Ética en enfermería en Colombia, período 2006-2010. Directora de postgrados, Fundación Universitaria de Ciencias de la Salud.
BLACKWELL SYNERGY, COCHRANE y VIREME con el propósito de buscar referencias de enfermería relacionadas con la temática, procedí a leer más de 380 resúmenes y después 69 artículos completos una vez realizada la selección.

Mientras realizaba el análisis de la revisión de literatura me encontré que seis de esos artículos expresan de diversas maneras que las enfermeras no cuidan a las personas ancianas, pues las marginan de la atención que brindan en los servicios enunciados por las investigadoras (medicina interna y urgencias). 
Además, tratan a la persona anciana con desprecio, la despersonalizan, son indiferentes con sus sensaciones, percepciones, necesidades y dolencias. Ante estos resultados me surgieron interrogantes sobre los conceptos que tienen las enfermeras respecto a la vejez para que actúen de esa manera: ¿Cómo concibe la enfermera la vejez y qué valor le da? ¿Será que las enfermeras menospreciamos lo que representa la vejez? ¿Será que para algunas enfermeras el anciano o viejo pierde la condición de persona? ¿Por qué razón somos indiferentes ante sus sensaciones, percepciones, necesidades y dolencias? ¿Conocemos, respetamos y valoramos el concepto que tienen los ancianos sobre la vida y sus prácticas de salud? Esto me permitió considerar que era pertinente realizar el ejercicio académico de aclarar el concepto de vejez, ya que como dice E. de Reales "de la abundancia del corazón hablan los actos y las palabras de cuidado", es decir, como concebimos la vejez ¿así actuamos frente a ella?

Los artículos restantes de la revisión hecha abordan en forma indiscriminada el concepto de vejez con los del viejo envejecido, anciano (aged, enderly), persona mayor (older people) o adulto mayor lo cual permite ver que no hay claridad en el uso del concepto por parte de enfermería, sobre todo en el escenario hospitalario.

\section{Selección del concepto}

A través del presente ejercicio se analizará el concepto de vejez, que como se expresó en la presentación, estuvo influido por la revisión de la literatura y el desarrollo de la autora como enfermera de servicio y como docente.

\section{Identificación del concepto}

Teniendo como marco de referencia la revisión de la literatura que evidencia poca claridad en la concepción de vejez por parte de enfermería y a la luz del incremento en la esperanza de vida en la población colombiana, latinoamericana y mundial, se plantea la necesidad de estudiar y comprender la vejez y el proceso de envejecimiento. Los datos encontrados en la revisión de la literatura corresponden a la descripción desde la antropología, sociología, medicina y enfermería de lo que es ser viejo física, mental, emocional y socialmente; nos presentan también la visión sobre cómo se perciben los viejos y como lo hacen sus familias y el equipo de salud.

\section{Análisis del concepto}

1.Objetivo del análisis: clarificar el concepto de vejez, que incluye aspectos sobre cómo y cuándo se usa y qué significado tiene para las enfermeras.

Los objetivos específicos corresponden a:

- Desarrollar una definición operacional de vejez.

- Determinar la definición de los atributos críticos del concepto.

- Identificar las referencias empíricas para los atributos críticos del concepto de vejez.

\section{Usos del concepto de vejez}

a) Uso en teorías del comportamiento humano: se refiere al proceso de envejecimiento primario como son los cambios graduales e inevitables relacionados con la edad, que aparecen en todos los miembros de una especie. Es normal y sucede a pesar de la salud, el estilo de vida activo y carecer de enfermedades. Compartiremos algunas teorías. Se subraya el concepto con el cual se denomina la vejez dentro de cada una de las teorías del comportamiento humano.

Teoría psicosocial de Ericsson: cuando los adultos entran en la etapa final de la vida, dijo Ericsson, la tarea consiste en contemplar su vida en conjunto y con coherencia. Necesitan aceptar su propia vida tal como la han vivido y creer que lo hicieron lo mejor posible en sus circunstancias. Si tienen éxito en esta tarea, habrán desarrollado la integridad del ego, que sólo puede obtenerse tras haber luchado contra la desesperación. Cuando esta domina, la persona teme la muerte y aunque pueda expresar desprecio 
por la vida, continúa anhelando la posibilidad de volver a vivirla. Cuando impera la integridad, la persona posee la fuerza propia de su edad, que es la sabiduría. Con ella el adulto (viejo) sabe aceptar las limitaciones. El adulto sabio (viejo) sabe cuándo aceptar el cambio y cuándo oponerse al mismo, cuándo sentarse en silencio y cuándo luchar.

El paso a la tercera edad no pone fin a la generatividad (generación de ideas y procesos) de una persona. A medida que la categoría de las personas mayores aumenta, Erikson predice que éstas permanecerán implicadas en los asuntos del mundo durante mucho más tiempo. Contempla la tercera edad como un período más productivo y creativo que lo aceptado en el pasado, de manera que un artista de 80 años, escritor o músico, ya no será algo excepcional.

En su crisis final, integridad vs. desesperación, Erikson considera que "las personas de edad avanzada enfrentan una necesidad de aceptar su vida -la manera como han vivido- con el fin de aceptar su muerte próxima. Luchan por lograr un sentido de integridad, de coherencia y totalidad de la vida, en vez de dar vía a la desesperación sobre la incapacidad para volver a vivirla de manera diferente. Las personas que han tenido éxito en esta tarea integradora final construyendo sobre los resultados de las siete crisis previas, ganan un sentido más amplio del orden y del significado de sus vidas dentro de un ordenamiento social más grande, pasado, presente y futuro. La 'virtud' que se desarrolla durante esta etapa es la sabiduría, un despreocupado e informado interés por la vida de cara a la muerte en sí misma"; incluye aceptar lo vivido, sin arrepentimientos importantes sobre lo que pudo haber sido diferente, implica aceptar a los padres como las personas que hicieron lo mejor que podían y por tanto merecen amor, aunque no fueran perfectos, y aceptar la propia muerte como el fin inevitable de la vida. Propone que las personas que no consiguen aceptar esto son desesperanzadas, porque ya no ven tiempo para reaccionar y enmendar aquello con lo que no están de acuerdo.
Continúa Erikson "la edad adulta tardía es también una época para jugar, para rescatar una cualidad infantil esencial para la creatividad. El tiempo para la procreación ha pasado, pero la creación puede tener lugar todavía. Aun a medida que las funciones del cuerpo se debilitan y la energía sexual puede disminuir, las personas logran disfrutar una experiencia enriquecida, corporal y mentalmente".

La teoría de Jung vió como las personas mayores todavía se esforzaban para desarrollarse a sí mismas, dado que creía que rara vez se alcanzaba una personalidad integrada. Dentro de cada individuo veía fuerzas y tendencias en conflicto que necesitaban ser reconocidas y reconciliadas. Parte de este reconocimiento se refleja en la tendencia de cada género a expresar rasgos por lo regular asociados con el otro sexo. Proponía que esta tendencia aparecía por primera vez en la mitad de la vida y observó que la expresión del potencial de género que estaba oculto aumentaba en la tercera edad. Durante esta, la expresión de los hombres sobre su feminidad y la de las mujeres de su masculinidad, supone otro intento de reconciliar las tendencias en conflicto. Anotó que dentro de cada persona existía una orientación hacia el mundo exterior, que denominó extroversión, y otra al interior, el mundo subjetivo, que llamó introversión. En la juventud y gran parte de la mediana edad, las personas expresan su extroversión. Una vez que la familia ya ha salido adelante y la vida profesional toca a su fin, hombres y mujeres se sienten libres para cultivar sus propias preocupaciones, reflexionar sobre sus valores y explorar el mundo interior. "Para una persona joven", escribió Jung “es casi un pecado o al menos un peligro preocuparse por ella misma; pero para la persona que está envejeciendo, es un deber y una necesidad dedicar seria atención a sí misma". Este cambio de orientación conduce a las personas mayores a desarrollar con paso firme la tendencia hacia la introversión.

Pensamiento pos-formal de Jean Piaget: consideró que el elemento más importante del pensamiento eran las operaciones lógicas. No obstante, el pensamiento del adulto es flexible, abierto y puede adaptarse de 
muchas maneras que van más allá de la lógica abstracta, lo cual se remite -en ocasiones- al pensamiento pos-formal. La madurez del pensamiento se refleja en la capacidad de combinar lo objetivo (elementos lógicos o racionales) con lo subjetivo (concretos o basados en la experiencia personal). Esto ayuda a que las personas tengan en cuenta sus propias vivencias y sentimientos En este momento puede aflorar la sabiduría como pensamiento flexible que capacita a la personas para aceptar la inconsistencia, la contradicción, la imperfección y el compromiso, de manera que puedan resolver problemas de la vida real.

Esta madurez de pensamiento, o pensamiento posformal, se basa en la subjetividad, la intuición y la lógica pura, característica del pensamiento en las operaciones formales. Los pensadores maduros personalizan su razonamiento y emplean la experiencia cuando tienen que enfrentarse a situaciones ambiguas. El pensamiento pos-formal también se caracteriza por un desplazamiento de la polarización (correcto vs. incorrecto, lógica vs. emoción, mente vs. cuerpo) hacia una integración de conceptos.

b) Uso del concepto de vejez en enfermería. Se puede expresar que dentro de las teóricas es Dorotea Orem quien aborda en forma directa el concepto y lo incluye en la exposición de uno de los supuestos humanos, en donde define: "los seres humanos maduros experimentan privaciones en forma de limitaciones de la acción en el cuidado de sí mismos y de los demás, haciendo de la sostenibilidad de vida los estímulos reguladores de la función".

Fawcett al finalizar cada capítulo analiza y evalúa los modelos conceptuales y las teorías, y compila las publicaciones e investigaciones que se han realizado dentro del marco de cada modelo conceptual o teoría de enfermería. En todas se encuentran estudios relacionados con las diferentes etapas de la vida y sujetos de atención y dentro de ellas por supuesto se aborda la vejez.

Según Kim, Castrillón y cols. y Jiménez y cols. las enfermeras dentro de los procesos de investigación han incluido desde mediados del siglo pasado el estudio de la vejez, sus características, causas y consecuencias, pero se evidencia el uso indistinto de los términos vejez, ancianidad, tercera edad, edad madura y persona anciana, al igual que en la revisión de la literatura realizada para el presente ejercicio. Con el fin de identificar como perciben y usan las enfermeras el concepto de vejez, se elaboró una sencilla y rápida encuesta por internet, la cual fue enviada a 26 enfermeras con experiencia superior a quince años, todas ellas además maestras a quienes se les solicitó que respondieran estos enunciados:

- ¿Qué es para usted la vejez?

- ¿Cómo usa este término y con que sinónimos los relaciona?

- ¿Cuándo usa el concepto, en qué momentos de su expresión verbal o escrita?

Los resultados encontrados en los diez correos que respondieron están sintetizados en la Tabla 1. De las respuestas presentadas por las enfermeras la vejez es una etapa de la vida (90\%), los deterioros pueden o no estar presentes (todo depende de la calidad de vida, $40 \%$ ), tiene que ver con la edad cronológica de 60 años (20\%), permite y evidencia el desarrollo personal que depende del contexto y la experiencia de vida que se haya tenido $(30 \%)$, en la medida que las personas se acercan a esta etapa la miran con mayor amplitud, respeto y proposición (30\%). Todas las anteriores respuestas están relacionadas en forma coherente con la literatura, excepto la última respuesta.

En cuanto a los sinónimos, existe una variedad mayor a la presentada por la literatura y tiene que ver con la cultura costumbrista que presenta el país en sus diferentes regiones. Las enfermeras enuncian otros términos para referirse a la vejez que yo no conocía. Y en relación al uso del concepto, el más frecuente es como adjetivo calificativo (positivo o negativo) para denominar a una persona cariñosa o peyorativamente, incluso cuando es joven. 


\section{Tabla I. Síntesis de las respuestas}

PREGUNTA

\section{RESPUESTA}

\begin{tabular}{|c|c|}
\hline ¿Qué es para usted la vejez? & $\begin{array}{l}\text { La vejez comienza a partir de los } 60 \text { años, es una etapa de la existencia cuando se } \\
\text { alcanza madurez en todos los aspectos y en la cual nuestras vidas cambian y se } \\
\text { tornan más lentas, más profundas. El envejecimiento depende de la calidad de vida de } \\
\text { las personas, la cultura, el contexto socioeconómico, el rol que se desempeñe en la } \\
\text { familia y en la sociedad; todos los días se envejece. }\end{array}$ \\
\hline $\begin{array}{l}\text { ¿Cómo usa este término } \\
\text { y con qué sinónimos los } \\
\text { relaciona? }\end{array}$ & $\begin{array}{l}\text { - Para referirse a personas familiares, de lo contrario don o doña. } \\
\text { - El término cuando se usa en el medio social, productivo y laboral cuando concen- } \\
\text { tra la mayor carga negativa; en el medio político y en el cultural tiene múltiples } \\
\text { acepciones. } \\
\text { - En salud tiene interpretaciones y abordajes desde lo biológico, psicológico y } \\
\text { social. } \\
\text { Sinónimos: catano, senil, achacoso, veterano, arcaico, longevo, ochentón, entrado } \\
\text { en años, traqueado, anciano, tener alto kilometraje, tener sus abriles, baquiano, } \\
\text { chuchumeco, viejo, envejecido, oxidado, ancianidad, senectud, decrépito. En el ámbito } \\
\text { de la salud se utilizan mucho los términos de anciano, geronte, viejo. }\end{array}$ \\
\hline $\begin{array}{l}\text { ¿Cuándo lo usa, en qué } \\
\text { momentos de su expresión } \\
\text { verbal o escrita? }\end{array}$ & $\begin{array}{l}\text { - Al saludar o al despedirse; cuando me refiero a las personas mayores de } 65 \text { años; } \\
\text { es una costumbre familiar para llamar a mis viejitos (mamá y papá). } \\
\text { - Para referirme a un grupo de personas con las que tengo nexos de trabajo (mis } \\
\text { viejitos de investigación). } \\
\text { - Como admiración y ternura "tan linda la viejita o el viejito...tiene cara de } \\
\text { muñeca". } \\
\text { - Yo utilizo en el ámbito profesional los términos anciano, anciana, viejo, vieja y en } \\
\text { el afectivo los apelativos de cuchita, mi viejita... En muchas oportunidades utilizo } \\
\text { la palabra vieja o viejo al referirme a personas a las que profeso afecto pero que } \\
\text { no siempre son ancianas. }\end{array}$ \\
\hline
\end{tabular}

c) Definición de vejez: para concretar el término vejez, se analizaron varias definiciones incluidas las reportadas por la revisión de la literatura consultada. Se encontraron algunas similitudes y las diferencias fueron más indeterminaciones respecto a la concepción y uso del término. Algunas de esas varían según el área social y de la salud.

Para la O.M.S. la edad media se extiende de los 45 a los 59 años, la de las personas de edad avanzada de los 60 a los 74 años, la de los ancianos de 75 hasta los 90 años, considerándose como grandes ancianos a las personas de más de 90 años. En las concepciones médico-sociales actuales se suele hablar de "tercera edad" a partir de los 65 años, edad de la jubilación y de la "cuarta edad" a partir de los 80 años. Pero estas cifras tienen un valor muy relativo, ya que todo depende de los procesos de senectud biológica y psicológica."

Prevalece la noción de que la vejez es resultado inevitable del deterioro orgánico y mental que se hace visible a mediados de la vida. De ahí en adelante, progresa a un ritmo acelerado. La vejez o adultez mayor es la cualidad de ser viejo. A las personas se les dice viejas porque en general han vivido más tiempo que las demás. Sin embargo, la definición depende del contexto y del grupo de personas al que se refiera. 
En uno de los estudios las enfermeras describen la vejez a partir de los 60 años, otros a partir de 65, en ambos casos los procesos físicos, psicológicos, mentales, afectivos y sociales dependen del contexto en el que se encuentre enmarcada la persona, las condiciones y la calidad de vida.

Como definición extraída de la revisión de la literatura el concepto de anciano, adulto mayor, viejo se refiere a personas que se encuentran en edad igual o superior a los 65 años para Colombia, América Latina y el contexto mundial en general. Lo común en la definición independiente de la fuente de información consultada, es la evidencia de indicadores como edad cronológica y factores categorizantes como condición de existencia, calidad, estilos de vida y hábitos (nutricionales, de actividad física y mental, sociales y culturales, entre otros). El concepto de envejecimiento está expresado como esa transformación biológica progresiva que se genera en el cuerpo y que dependiendo de los hábitos, estilos y calidad de vida del sujeto, compromete su salud o favorece la aparición de enfermedades agudas o crónicas que inician un ciclo sin fin de incapacidades e inhabilidades personales, familiares y sociales. Es percibida de diferentes formas tanto por el anciano mismo como por parte del personal de enfermería, la familia y los estudiantes de enfermería. Dentro de esas percepciones están la decadencia, degeneración, pérdida, senectud y caducidad.

Otro elemento relacionado con la vejez es la calidad de vida, que de acuerdo con la revisión de literatura corresponde al bienestar, felicidad y satisfacción de la persona que le permite una capacidad de actuación o de funcionar en un momento. Es un concepto subjetivo, propio de cada individuo, que está muy influido por el entorno en el que vive como la sociedad, la cultura y las escalas de valores. El Banco Mundial lo define como el bienestar general de la población. La calidad de vida es difícil de medir (sea para un individuo, un grupo o una nación) porque, además del bienestar material, incluye componentes intangibles como la calidad del medio ambiente, la seguridad nacional, la personal y las libertades políticas y económicas.
Concebida también como el grado de satisfacción de las necesidades de las personas o de los grupos sociales, se refiere al conjunto de condiciones que definen la situación y el estado de una población humana en su medio. Por último, es el bienestar físico, mental, ambiental y social como es percibido por cada individuo y cada grupo. Dependen también de las características del medio ambiente en que el proceso tiene lugar (urbano, rural).

Aumento de la esperanza de vida: concepto abordado en algunos de los artículos, corresponde al incremento de la relación entre todos los años vividos por toda la población sobre los niños nacidos en un año; también definido como el número de años de vida que restan, término medio, a una persona de no variar la tendencia de la mortalidad. Se cita por lo regular como esperanza de vida al nacer. Al unir varias definiciones, se entiende como la cantidad promedio de años que se espera que una persona viva a partir de su nacimiento.

El estilo de vida se define según la epidemiología como el hábito y la forma de existencia que comprende el conjunto de comportamientos o actitudes que desarrollan las personas, unas veces saludables y otras nocivas para la salud. En los países desarrollados los estilos de vida poco saludables son los que causan la mayoría de las enfermedades. Dentro del triángulo epidemiológico causante de estas, estaría incluido el factor huésped. También es definido el estilo de vida como la que nace de las necesidades, percepciones, actitudes, intereses y opiniones individuales. El hábito de vida tiene que ver con la ejecución frecuente de una acción; se acostumbra por aprendizaje, rutina y repetición a hacer algo.

A continuación se definen la vejez y algunos conceptos relacionados con ella, expresados en el diccionario virtual de Encarta, autorizado por la Real Academia de la Lengua Española:

Vejez f. Cualidad de viejo, edad senil, senectud; achaques, manías, actitudes propias de la edad de los viejos. Dicho o narración de algo muy sabido y vulgar. 
Viejo, ja. (Del lat. vulg.věclus, y este del lat. vetŭlus) adj. Se dice de la persona de edad. Comúnmente puede entenderse que es vieja la que cumplió 70 años, antiguo o del tiempo pasado.; que no es reciente ni nuevo. Ser viejo en un país. Deslucido, estropeado por el uso. m. y f. coloq. Am. U. como apelativo para dirigirse a la madre o al padre, a la esposa o al esposo, o entre amigos.

Anciano, na. (Del lat. *antiānus, de ante). adj. Dicho de una persona: de mucha edad. Antiguo, que existe desde hace tiempo.

Senil. (Del lat. sen̄̄lis) adj. Perteneciente o relativo a la persona de avanzada edad en la que se advierte su decadencia física.

Con todo lo anterior, defino entonces la vejez como la existencia de una etapa o ciclo en la vida de una persona que corresponde a una ubicación cronológica a partir de los 65 años de edad, cuyo proceso multifactorial tiene lugar durante la última etapa del ciclo vital y que se caracteriza por la disminución progresiva de la capacidad funcional en todos los tejidos y órganos del cuerpo y de la consiguiente habilidad para ajustarse a estímulos ambientales. Debido a esto el anciano puede percibir sentimientos de frustración, tristeza o aislamiento al no poder cumplir de la misma manera su rol familiar o social; como consecuencia de factores particulares de hábitos, estilos y calidad de vida, se puede evidenciar o no la presencia de enfermedades crónicas.

\section{Atributos críticos del concepto de vejez: son} aquellos que se encuentran en forma repetida durante la revisión de la literatura. La lista ayuda a nombrar la ocurrencia de un fenómeno específico, cómo diferenciarlo de otros similares o uno relacionado. Los atributos más frecuentes fueron:

- Ubicación cronológica a partir de los 65 años de edad.

- Causas de vejez conocidas y descritas desde diferentes disciplinas de la salud y de las ciencias sociales.
- Percepciones y respuestas de los ancianos frente a su condición física, mental, emocional, espiritual, familiar y social de salud y vida, las cuales se encuentran descritas en documentos y mediante investigaciones.

- Sentimientos de frustración, decepción, tristeza, melancolía, abandono o aislamiento social, inhabilidad funcional física y familiar de acuerdo a como se ven, se sienten y son tratados inclusive por el personal de salud.

Referencias empíricas del concepto de vejez: tenemos la descripción de los atributos del concepto de vejez (Tabla 2), unos objetivos y otros subjetivos. Dentro de los objetivos, el primero es la referencia cronológica a partir de los 65 años, el segundo es una etapa conocida y documentada del ciclo vital humano, es un proceso influido por múltiples factores y que compromete la disminución progresiva de la capacidad física, mental, espiritual, social, familiar, etc. Desde lo subjetivo el anciano se percibe despersonalizado ya que parece que frente a los ojos de los demás pierde su propia identidad, es mirado con indiferencia, como si no existiera; es inhabilitado al tornarse inseguro, lento o torpe en el desarrollo de sus movimientos y acciones. Todo esto lo lleva a ser despojado de su rol y función familiar y social.

a) Antecedentes del concepto: cuando se busca la definición y evolución del concepto de vejez y el proceso de envejecimiento referido a seres vivos en documentos, investigaciones, diccionarios y enciclopedias, suele referirse a los aspectos biológicos. Así, la vejez es la calidad de viejo, asociada al deterioro físico y psíquico, y se refiere al último período del ciclo vital. Si se reúnen las distintas definiciones, desde el punto de vista biológico la vejez aparece como resultado de un proceso, entendiéndolo como el conjunto de modificaciones inevitables e irreversibles que se producen en los seres vivos cuyo final es la muerte.

Pero una cosa es la definición de la vejez y otra su conceptualización, lo que significa para nosotros y lo asociado con este término. Es aquí donde encontramos una gran diversidad y relatividad en cuanto 


\begin{tabular}{|c|c|}
\hline Subjetivas & Objetivas \\
\hline $\begin{array}{l}\text { Despersonalizado. } \\
\text { Tratado con indiferencia. } \\
\text { Anulado del contexto social, familiar, etc. } \\
\text { Inhabilitado. } \\
\text { Despojado de su rol familiar y social. } \\
\text { Relegado de su función familiar y social. }\end{array}$ & $\begin{array}{l}\text { Edad cronológica a partir de los } 65 \text { años. } \\
\text { Proceso de envejecimiento influido por múltiples factores. } \\
\text { Genera disminución progresiva de las capacidades física, mental, } \\
\text { emocional, espiritual, familiar, social, etc. } \\
\text { Puede presentar o no enfermedad crónica. } \\
\text { Existe variación en los signos vitales que se agudizan si existen } \\
\text { patologías de base que comprometan la circulación o la respiración. } \\
\text { Incontinencia urinaria. } \\
\text { Disminución de la fuerza muscular y la resistencia física. } \\
\text { Disminución de la agudeza visual. } \\
\text { Disminución de los reflejos y la coordinación. } \\
\text { Disminución de los procesos de sueño. }\end{array}$ \\
\hline
\end{tabular}

a elementos y concepciones, ya que el proceso de envejecimiento no es igual para todos los seres humanos ni en todas las épocas históricas. Recuerdo el capítulo de un libro medieval que empezaba diciendo algo así como: "había un viejo de pelo y barba blanca, de unos 40 años, sentado bajo un árbol". Creo que este es un buen ejemplo de cómo es relativo al tiempo, la época, el contexto, la cultura en que se vive y se desarrolla. En esta breve frase se puede apreciar muy bien la relatividad de los conceptos que se barajan al hablar de este tema. El fragmento que he citado corresponde a una época muy concreta, el período medieval, en el que las tasas de mortalidad eran muy elevadas y la esperanza de vida rondaba los 30 años, los pocos que la superaban eran considerados ancianos y, en cierto modo, sabios en virtud a los conocimientos acumulados a lo largo de su trayectoria vital.

En la actualidad, en países como España, que posee uno de los índices más elevados de esperanza de vida del mundo, 82 años para las mujeres y 75 años para los hombres, se consideran viejos a personas que están por debajo de la media (75 años). ¿A qué se debe esto? Son muchos los factores que influyen, no sólo aquellos demográficos y vitales, como el descenso de la tasa de mortalidad o el envejecimiento progresivo de la población.
Desde hace unas décadas se está engendrando un cambio en cuanto al concepto de ancianidad, así como al trato y consideración hacia estas personas. Del respeto y la deferencia en el pasado, al poseer el conocimiento, hoy día son tratados en muchas ocasiones como niños o menores de edad social porque "chochean", es decir, porque tienen sus facultades mentales disminuidas. Si bien ante esto uno se pregunta hasta qué punto esta reducción de su capacidad mental es consecuencia del proceso de envejecimiento y hasta dónde es debida a la falta de uso, por asumir ellos mismos las características que la comunidad adjudica a los ancianos. La sociedad y los medios de comunicación de masas los muestran jugando al dominó o a las cartas en los centros de jubilados o cuidando a los nietos como actividades "propias" de su edad mientras que se presentan como excepcionales, sin tener por qué serlas, la asistencia a centros de adultos, la utilización de nuevas tecnologías, la concurrencia a las salas de cine destinadas a la "tercera edad", cuyo único requisito para entrar es tener más de 65 años, o mostrar a los ancianos embarcados en diversas aventuras deportivas o intelectuales. De modo que no solo se les está negando la capacidad de sus facultades sino hasta la ilusión.

En la actualidad se considera a los ancianos como personas que no saben, se podría decir que se les 
asigna estatus carente de roles definidos en la sociedad contemporánea, desplazándolos también de este modo de los sistemas de control y de poder, que pasan a manos de los "jóvenes", a los que se atribuye mayores conocimientos académicos o técnicos en razón de los rápidos cambios que está viviendo nuestro mundo. Esto se comprueba en algunas de las investigaciones realizadas por las enfermeras.

b) Consecuencias: de la clarificación del concepto de vejez se debe establecer unidad en el uso clínico del término. Así mismo, debe permitir al equipo de enfermería recrear el conocimiento de lo que es y representa este sujeto de atención de cuidado y la etapa que cursa. En forma progresiva nos comprometeremos a crear estrategias de cuidado de enfermería que satisfagan las necesidades del anciano, su familia y la sociedad. Es oportuno y necesario para las enfermeras continuar desarrollando conocimientos en esta área del cuidado respecto a la presencia de viejos enfermos, pero también de viejos sanos, así como permitir la evolución del concepto y la caracterización del mismo en relación con lo que se vive en lo biosicosocial y cultural.

Otras consecuencias de conocer el concepto de vejez y sus dimensiones corresponde a la recuperación o mantenimiento de la confianza de la persona anciana y su familia en la atención de los servicios de salud. Esto facilitaría el abordaje de sus inquietudes, condiciones de vida, educación individual y familiar, aplicación de estrategias para la prevención de la enfermedad y sus complicaciones, así como la promoción de prácticas saludables.

\section{Conclusiones}

El objetivo propuesto para el presente documento corresponde a la clarificación del concepto vejez, cómo y cuándo se usa y qué significado tiene para las enfermeras. Se evidencia que en el ejercicio de la profesión hay una variada gama de expresiones, interpretaciones y usos. Es necesario en el ámbito clínico intrahospitalario, extramural y en el cuidado en casa unificar su definición, aplicación, intervención y uso.
Teniendo en cuenta que la sociedad es cambiante y dinámica y que a la par de los avances tecnicocientíficos también se trasforma el hombre, es necesario mantener el espíritu investigativo activo para detectar de manera oportuna esas variaciones en cada fase del ciclo de vida, en especial en la vejez. Comprensión que es importante para intervenir como profesionales de enfermería.

El incremento de la esperanza de vida a nivel local y mundial es un llamado para que las enfermeras nos interesemos en conocer y comprender la vejez y desde ese entendimiento y respeto proponer estrategias de cuidado que tengan sentido y valor para las personas ancianas, sus familias y por supuesto la sociedad en general.

Por último, quiero expresar cómo este trabajo resultó ser un ejercicio complejo, gratificante y trascendente para el manejo del lenguaje común y preciso en el ámbito clínico y disciplinar, y me permite realizar la invitación fraterna para que docentes, profesionales y estudiantes nos apropiemos correctamente de los conceptos y les demos el uso que corresponde al nivel de desarrollo académico.

\section{Lecturas recomendadas}

- Alabaster, Erica S. Students' ideals for nursing older people in practice. Int J Older People Nurs. 2006 Jun;1(2):6674.

- Bernis Cristina. Envejecimiento, poblaciones envejecidas y personas ancianas. Aging, aged populations and elderly people. Antropo [publicación seriada en Internet]. 2004 [citado 02 Feb 2008];6:[aprox. 14 p.]. Disponible en: http://www.didac.ehu.es/antropo/6/6-1/Bernis.pdf.

- Cutcliffe John R., McKenna Hugh P., The essential Concepts of nursing. Edinburgh: Elsevier, Churchil Livingstone; 2005.

- Cheek J, Ballantyne A, Roder-Allen G. Factors influencing the decision of older people living in independent units to enter the acute care system. J Clin Nurs. 2005 Mar;14 Suppl 1:24-33.

- Duran de Villalobos, María Mercedes. Enfermería: desarrollo teórico e investigativo. Bogotá: Universidad Nacional de Colombia; 2001. 
- Fawcett Jacqueline. Contemporary nursing knowledge: analysis and evaluation of nursing models and theories. 2nd. Ed. Philadelphia, FA, Davis Company, 2005.

- Fernández Larrea N, Clúa Calderón AM, Báez Dueñas RM, Ramírez Rodríguez S, Prieto Díaz V. Estilos de vida, bienestar subjetivo y salud de los ancianos. Rev Cubana Med Gen Integr. 2000;16(1):6-12.

- Fulton MM, Allen ER Polypharmacy in the elderly: a literature review. Journal of the American Acamedy of Nurse Practitioners. 2005, Apr; 17 (4) 123-132.

- Han Kwee H, Kozo M, Taizo W, Miyuki K, Toru K, Kiyofumi S. Factors associated with ADL dependence: A comparative study of residential care home and community-dwelling elderly in Japan. Geriatr Gerontol Int. 2002 Mar;2(2):80-6.

- Heikkilä K, Ekman SL. Health care experiences and beliefs of elderly Finnish immigrants in Sweden. J Transcult Nurs. 2000 Oct;11(4):281-9.

- Hodges HF, Keeley AC, Grier EC. Masterworks of art and chronic illness experiences in the elderly. J Adv Nurs. 2001 Nov;36(3):389-98.

- Kim MI. Nursing research: a worldwide picture. Rev Esc Enferm USP. 1992 Oct;26(Suppl):7-22.

- Meleis Afaf Ibrahim. Theorical nursing: development and progress. San Francisco: Lippincott Williams \& Wilkins, 1997.

- Olszewski Walker Lorraine, Coalson Avant Kay. Strategies for Theory Construction in Nursing, 4th. Ed. New Jersey: Prentice Hall; 2005.
- Reyes-Ortíz CA, Camacho ME, Eschbach K, Markides K. El contexto de la familia y el vecindario en la salud de los ancianos del estudio Epese hispano. Colombia Médica. [publicación seriada en Internet]. 2006 [citado $02 \mathrm{Feb}$ 2008];37(1):[aprox. 6 p.]. Disponible en: http://redalyc. uaemex.mx/redalyc/pdf/283/28337908.pdf.

- Rodríguez Mañas C. Atención domiciliaria para personas ancianas: no todo vale. Rev Esp Salud Publica. 2003 SepOct;5: 523-6.

- Roe B, Whattam M, Young H, Dimond M. Elders' needs and experiences of receiving formal and informal care for their activities of daily living. J Clin Nurs. 2001 May;10(3):389-97.

- Ross MM, Crook J. Elderly recipients of home nursing services: pain, disability and functional competence. J Adv Nurs. 1998 Jun;27(6):1117-26.

- Van Servellen G, Fongwa M, Mockus D'Errico E. Continuity of care and quality care outcomes for people experiencing chronic conditions: A literature review. Nurs Health Sci. 2006 Sep;8(3):185-95.

- Whetsell M, Fredirickson K, Aguilera P, Maya JL. Niveles de bienestar espiritual y de fortaleza relacionados con la salud en adultos mayores. Aquichan. 2005 Oct:5(1):7285.

- Wissing U, Ek AC, Unosson M. Life situation and function in elderly people with and without leg ulcers. Scand J Caring Sci. 2002 Mar;16(1):59-65.

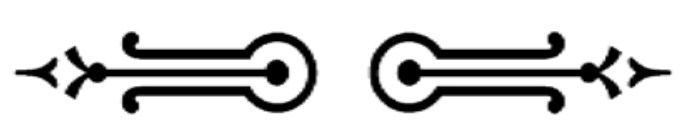

